\title{
Alterações Comunicativo-Cognitivo-Comportamentais Após Acidente Vascular Cerebral de Hemisfério Direito: Publicações Nacionais e Internacionais
}

\author{
Camila Rosa de Oliveira $^{* a}$, Gigiane Gindri ${ }^{a}$, Gabriela Damasceno Ferreira ${ }^{b}$ Francéia Veiga Liedtke $^{b}$, \\ Juliana de Lima Müller ${ }^{b}$, Thaís Ferrugem Sarmento ${ }^{b}$, Nicolle Zimmermann ${ }^{c}$, \\ Maria Alice de Mattos Pimenta Parente ${ }^{a} \&$ Rochele Paz Fonseca ${ }^{b}$ \\ ${ }^{a}$ Pontifícia Universidade Católica do Rio Grande do Sul, Porto Alegre, Brasil, bUniversidade Federal do Rio Grande do Sul, \\ Porto Alegre, Brasil \& ${ }^{c}$ Universidade do Rio dos Sinos, São Leopoldo, Brasil
}

\begin{abstract}
RESUMO
Este artigo visa traçar um panorama das publicações nacionais e internacionais quanto ao estudo das alterações comunicativo-cognitivo-comportamentais associadas as lesões vasculares de hemisfério direito. As bases LILACS e MEDLINE foram consultadas com as palavras-chave em português e em inglês: síndrome, hemisfério e direito. Os estudos foram selecionados a partir de critérios de inclusão. Encontraram-se poucos estudos que abordam essas alterações, todos internacionais, sendo as dificuldades cognitivas as mais descritas. Sequelas em todas as funções cognitivas e nos processamentos comunicativo e emocional são consideradas parte deste quadro. São indispensáveis estudos a respeito das alterações comunicativo-cognitivo-comportamentais pós-AVC de hemisfério direito para identificar diferentes perfis clínicos e contribuir para o aumento da eficácia dos procedimentos de avaliação e de reabilitação.
\end{abstract}

Palavras-chave: neuropsicologia; cognição; hemisfério direito; AVC.

\begin{abstract}
Communicative, cognitive and behavioral impairments associated with right hemisphere stroke: National and International Publications

The present paper aims to outline an overview of national and international publications concerning research on the communicative, cognitive and behavioral impairments associated with a right hemisphere stroke. Research on LILACS and MEDLINE databases were done using keywords in Portuguese and in English: syndrome, hemisphere and right for the search on the right hemisphere impairments. Studies were selected taking into account inclusion criteria. Only few studies about these disorders were found, all of them international investigations. Cognitive disorders were the most frequently described. Sequelae including all cognitive functions, emotional and communicative processing are considered part of this set of right hemisphere impairments. More studies about the RHS are necessary to identify different clinical profiles and increase the efficiency of assessment and rehabilitation process.
\end{abstract}

Keywords: neuropsychology; cognition; right hemisphere; CVA.

Classicamente, quando a função linguagem é abordada na literatura internacional e nacional, seus correlatos neurais incluem zonas corticais do hemisfério esquerdo (HE). A neuropsicologia surgiu justamente com a associação entre lesão no lobo frontal esquerdo e alterações linguísticas expressivas, com os casos clínicos apresentados por Paul Broca (1861), que estabeleceram o conceito de dominância hemisférica.
Essa noção preconiza que o hemisfério responsável pela linguagem é o dominante, em geral, o HE (Benedet, 1995; Martins, Antunes, Castro-Caldas, \& Antunes, 1995). Até a década de 1940, a relação HE e linguagem era praticamente exclusiva na literatura. No entanto, os pesquisadores da década de 1950 começaram a questionar os conceitos de "menor" e de "nãodominante", associados ao hemisfério direito (HD).

* Endereço para correspondência: Camila Rosa de Oliveira: oliveira.crd@ gmail.com 
Esse hemisfério, negligenciado até então, começou a ser considerado especializado na função perceptiva visual. Apesar de Jackson, em 1874 e 1876, já ter feito essa relação funcional, apenas 60 anos após, ela foi valorizada (Van Lancker, 1997).

No que diz respeito à linguagem, Eisenson (1959) associou pela primeira vez uma lesão de HD (LHD) a distúrbios linguísticos, há aproximadamente 50 anos. No entanto, apenas nas últimas duas décadas, os déficits comunicativos oriundos de uma LHD começaram a ser mais estudados com sistematicidade teórico-metodológica. Dessa forma, desde o final da década de 1980, a relação entre o HD e a função comunicativa vem sendo verificada em investigações com indivíduos neurologicamente preservados e com indivíduos que apresentam uma lesão nesse lado do cérebro, tanto em tarefas comportamentais como em avaliações com métodos de neuroimagem (Bouaffre \& Faïta-Ainseba, 2007; Brookshire, 2003; Code, 1987; Joanette, Goulet, \& Hannequin, 1990; Myers, 1999; Tompkins, 1995).

Atualmente, as alterações comunicativas, bem como as cognitivas e as emocionais, resultantes de um acometimento neurológico no HD têm sido abordadas na literatura. Esse conjunto de sinais e sintomas decorrentes de LHD é denominado por alguns autores como "Síndrome do Hemisfério Direito - SHD" (Blake, Duffy, Tompkins, \& Myers, 2003; Brookshire, 2003; Bryan, 1995; Kumral \& Evyapan, 2000; Landau, Auerbach, Gross-Tsur, \& Shalev, 2003; Marchetti, Carey, \& Della Sala, 2005; Morin e cols., 2001; Myers, 2001; Pimental \& Kingsbury, 1989). Uma das principais causas dessa síndrome é a lesão vascular (Tompkins, Fassbinder, Lehman-Blake, \& Baumgaertner, 2002). Entretanto, não há consenso quanto a nomenclatura deste quadro que ser caracteriza por um tripé que envolve (a) cognição, (b) linguagem/comunicação e (c) comportamento/emoção. As comunicativo-cognitivo-comportamentais pós-Acidente Vascular Cerebral (AVC) de HD se referem aos aspectos neuropsicológicos ligados a funções atencionais, mnemônicas, executivas, dentre outras, ao processamento de componentes verbais e não verbais que visam a uma meta comunicativa, por componentes fonológicos, morfológicos, léxico-semânticos, sintáticos, pragmáticos, prosódicos, e aos aspectos de ordem afetiva e psicoemocional.

As sequelas comunicativas observadas estão presentes principalmente em quadros de lesão vascular de $\mathrm{HD}$, com destaque para déficit na compreensão e na produção do discurso, no processamento de inferências e de informações contextuais, na interpretação de significados ambíguos e na compreensão e produção prosódica. Por conseguinte, pode-se observar que indivíduos com LHD podem apresentar alterações nos quatro processamentos comunicativos: discursivo, pragmático-inferencial, léxico-semântico e prosódico (Joanette e cols., 1990).

No que concerne às alterações cognitivas, estão presentes distúrbios nas funções de orientação têmporo-espacial, atenção, percepção, memória visual, habilidades aritméticas, praxias construtivas (Brookshire, 2003; Blake e cols., 2003) e nas funções executivas (Annoni e cols., 2003). Quanto aos aspectos emocionais, podem ser detectados os seguintes sintomas: déficits de percepção e de expressão das emoções transmitidas pela mímica facial, prejuízo na compreensão e na expressão de estímulos prosódicos com entonação emocional e distúrbios neuropsiquiátricos, como confusão, estados de paranoia, delírio, alucinações visuais, agitação, síndromes de identificação inadequada, mania secundária, dentre outros (Allegri e cols., 1996; Burns, Halper, \& Mogil, 1985; Myers, 1999).

É importante que investigações teóricas e empíricas envolvendo estudos com indivíduos normais e com LHD continuem sendo efetuadas para que neuropsicólogos clínicos e pesquisadores possam conhecer mais profundamente o papel do HD nas funções cognitivas, comunicativas e emocionais. Há importante lacuna quanto à estimativa de estudos de incidência de alterações comunicativo-cognitivo-comportamentais pós-AVC em decorrência das dificuldades metodológicas e, especialmente, no que se refere à terminologia. Entretanto, apesar da ausência de dados epidemiológicos, pelo menos metade dos pacientes com LHD apresentam distúrbio comunicativo, com manifestação heterogênea (Joanette e cols., 2007), podendo chegar a $92,40 \%$ com alterações quantitativas e/ou qualitativas nas habilidades comunicativas (Fonseca \& Parente, 2007).

Nesse contexto, o presente estudo tem como objetivo traçar um panorama das publicações nacionais $\mathrm{e}$ internacionais quanto ao estudo das alterações das funções dos processamentos cognitivo, comunicativo e emocional presentes nesta patologia neuropsicológica em adultos que tiveram AVC de HD. Para tanto, procurar-se-á responder às seguintes questões de pesquisa:

- Quantos estudos nacionais e internacionais fazem referência a alterações comunicativocognitivo-comportamentais lesão vascular de HD e como o fazem? 
- Quantas investigações nacionais e internacionais apresentam como objeto de estudo alterações nos processamentos de orientação têmporo-espacial, atenção, percepção, memória, habilidade aritmética, praxias, funções executivas, comunicação e emoção ligados à AVC de HD?

- Quais as alterações mais associadas à LHD após AVC?

\section{MÉTODO}

Para responder à primeira questão de pesquisa, realizou-se uma busca sistemática na base de publicações LILACS, para contemplar a literatura nacional, e MEDLINE, para a literatura internacional. Na base LILACS, utilizou-se o formulário livre sem limitação de data, com as seguintes palavras-chave em português: "síndrome AND hemisfério AND direito". Na base MEDLINE, usou-se o formulário livre de 1997 a 2009, com as palavras-chave em inglês: "right AND hemisphere AND syndrome". Esse termo foi utilizado por ser corrente na década de 2000.

Quanto às segunda e terceira questões de pesquisa, as mesmas bases de indexação foram utilizadas. Com o intuito de buscar as alterações cognitivas associadas à LHD, lançaram-se, na base LILACS, as seguintes palavras-chave em português: "orientação têmporoespacial", "atenção", "percepção", "anosognosia", "heminegligência", "memória", "habilidade aritmética", "matemática" e "funções executivas". Além dos termos que nomeiam cada função neuropsicológica, as palavras "anosognosia" e "heminegligência" também nortearam a busca, na medida em que são quadros muito frequentes pós-AVC de HD (Myers, 1999). Para encontrarem-se estudos sobre as alterações comunicativas, foram lançadas as palavras-chave "comunicação" e "linguagem". Na busca das alterações emocionais, as palavras-chave foram "emoção" e "comportamento". Todas essas palavras foram associadas à expressão "hemisfério direito". Em inglês, na base MEDLINE, foram lançadas as palavras-chave "time space orientation", "attention", "perception", "anosognosia", "neglect", "memory", "arithmetics", "mathematics" e "executive functions", na busca por alterações cognitivas. Para encontrarem-se estudos sobre alterações comunicativas, utilizaram-se as palavras "communication" e "language". Por fim, para as alterações emocionais, "emotion" e "behavior". Cada uma das palavras foi combinada com a expressão "right hemisphere". As buscas concernentes às questões de pesquisa foram realizadas em fevereiro de 2010 e incluíram estudos publicados até dezembro de 2009.
Os critérios de inclusão dos estudos encontrados pela busca inicial com as palavras-chave acima foram os seguintes: consistir em um artigo que aborde as alterações comunicativo-cognitivo-comportamentais ou sintomas relacionados a sequelas de AVC de hemisfério direito, podendo ser uma revisão teórica, um estudo de caso ou um estudo empírico com grupo de adultos com LHD; ter como participantes exclusivamente adultos; e, incluir indivíduos com LHD com acometimento neurológico caracterizado por AVC, hemorrágico ou isquêmico. Foi excluída, por exemplo, uma grande quantidade de publicações com indivíduos neurologicamente preservados; crianças e adolescentes com LHD; indivíduos com LHD com quadros neurológicos além do AVC, tais como tumores, traumatismos crânio-encefálicos, epilepsia, entre outros; associação de sintomas de LHD com outras patologias, tais como esquizofrenia, transtorno do déficit de atenção e hiperatividade, autismo, depressão, demência do tipo Alzheimer, entre outras. Desse modo, foram lidos e analisados todos os resumos e abstracts resultantes da busca inicial e foram mantidos na quantificação final aqueles que se enquadravam nos critérios de inclusão acima mencionados. Na busca com as palavras chaves em que as buscas iniciais resultaram em mais de 100 artigos, a pesquisa foi refinada acrescentando-se o termo "déficit", em português, e "deficit", em inglês, às palavras-chave iniciais, uma vez que as alterações comunicativo-cognitivo-comportamentais pós-AVC de HD necessariamente estão associadas a um prejuízo em alguma das funções estudadas.

\section{RESULTADOS}

Primeiramente serão apresentados os dados encontrados na busca nas bases LILACS e MEDLINE para obter um panorama nacional e internacional, respectivamente, da quantidade de estudos que fazem referência às alterações comunicativo-cognitivo-comportamentais pós-AVC de HD e como esses o fazem, ou seja, os resultados referentes à primeira questão de pesquisa. Na Tabela 1, pode ser observado o número de estudos nacionais e internacionais encontrados após a busca inicial com as palavras-chave "síndrome AND hemisfério AND direito", em português (LILACS) e inglês (MEDLINE). Além disso, também pode ser visualizado o número de investigações que resultaram da análise dos resumos e abstracts obtidos na busca inicial, isto é, número final de investigações pós-análise dos critérios de inclusão mencionados na seção Método. 
Tabela 1

Quantidade de Estudos Nacionais e Internacionais que Abordam as Alterações comunicativo-cognitivo-comportamentais pós- $A V C$ de HD

\begin{tabular}{ccc}
\hline Bases de dados & $\begin{array}{c}\text { Número de estudos } \\
\text { busca inicial }\end{array}$ & $\begin{array}{c}\text { Número de estudos pós-análise } \\
\text { critérios inclusão }\end{array}$ \\
\hline LILACS & 8 & 00 \\
MEDLINE & 377 & 31 \\
\hline Total & 461 & 31 \\
\hline
\end{tabular}

Com base na Tabela 1, observa-se que não foram incluídos estudos publicados na língua portuguesa após a análise dos critérios de inclusão. Das pesquisas publicadas em inglês encontradas na busca inicial, somente $8,22 \%$ foi mantida na quantificação final.

Na Tabela 2, os 31 estudos mantidos após a análise dos 377 abstracts encontrados no MEDLINE foram distribuídos quanto ao tipo de estudo - revisão, estudo de caso ou estudo de grupo. Também foram agrupados quanto ao objeto de estudo: as alterações comunicativo-cognitivo-comportamentais como um todo, englobando todos os sinais e sintomas após uma LHD; ou algum sintoma específico apenas, tais como as dificuldades de atenção e/ou percepção que consistem apenas em parte dos déficits que podem ocorrer após uma lesão vascular de HD.

Tabela 2

Distribuição das Publicações Encontradas na Base MEDLINE por Tipo e Objeto de Estudo

\begin{tabular}{ccccc}
\hline Objeto de estudo & \multicolumn{3}{c}{ Tipo de estudo } & Total \\
\cline { 2 - 5 } & Revisão & Estudo de caso & 00 & Estudo de grupo \\
\hline Déficits neuropsicológicos gerais pós-LHD & 01 & 02 & 17 & 28 \\
\hline Sintoma específico & 09 & 02 & 17 & 31 \\
\hline
\end{tabular}

Evidencia-se, a partir da Tabela 2, que do total de estudos de revisão, $10 \%$ apresentou como objeto de estudo os déficits neuropsicológicos gerais decorrentes de AVC de HD e $90 \%$, algum sintoma específico desse quadro. Da quantidade final de estudos de caso, $50 \%$ abordou as alterações comunicativo-cognitivo-comportamentais pós-LHD de forma geral, enquanto $50 \%$ enfocou algum sintoma específico. Dos estudos de grupo, $100 \%$ teve como objeto de estudo algum sintoma específico. Além disso, analisando-se a distribuição dos 31 estudos por tipo de pesquisa, constata-se que as investigações encontradas que abordavam as alterações pós-LHD propriamente dita ou os sinais e sintomas que a compõem foram predominantemente estudos de grupo $(54,84 \%)$ e estudos de revisão $(32,26 \%)$; os estudos de caso foram a minoria $(21,90 \%)$.
A Tabela 3 possibilita a visualização dos resultados referentes à segunda e à terceira questões de pesquisa. Expõe a quantidade de estudos nacionais - LILACS - e internacionais - MEDLINE - sobre alterações cognitivas, comunicativas e emocionais oriundas de uma LHD, por palavras-chave. Podem ser observadas duas quantificações dos estudos buscados na base LILACS: quantidade inicial de publicações e, após a análise dos critérios de inclusão, quantidade final de publicações. Em relação às investigações internacionais da base MEDLINE, três quantificações podem ser observadas: inicial, com todos os estudos encontrados; refinada, com o acréscimo da palavra-chave "déficit" em uma segunda etapa da busca nessa base; e, final, com o número final de estudos mantidos que se enquadravam nos critérios de inclusão. 
Tabela 3

Quantidade de Estudos Nacionais e Internacionais que Abordam Alterações do Processamento Cognitivo, Comunicativo e Emocional após LHD

\begin{tabular}{|c|c|c|c|c|c|}
\hline \multirow[t]{2}{*}{ Palavras-chave } & \multicolumn{2}{|c|}{$\begin{array}{c}\text { Quantidade } \\
\text { publicações } \\
\text { LILACS }\end{array}$} & \multicolumn{2}{|c|}{$\begin{array}{c}\text { Quantidade publicações } \\
\text { MEDLINE }\end{array}$} & \multirow[t]{2}{*}{ Final } \\
\hline & Inicial & Final & Inicial & Refinada & \\
\hline Anosognosia AND HD & 0 & 0 & 40 & - & 21 \\
\hline Atenção AND HD & 4 & 0 & 726 & 119 & 25 \\
\hline Comportamento AND HD & 2 & 0 & 247 & 25 & 7 \\
\hline Comunicação AND HD & 5 & 2 & 147 & 8 & 1 \\
\hline Emoção AND HD & 0 & 0 & 203 & 14 & 3 \\
\hline Funções executivas AND HD & 0 & 0 & 41 & - & 5 \\
\hline Habilidades aritmética AND HD & 0 & 0 & 1 & - & 0 \\
\hline Heminegligência AND HD & 3 & 3 & 443 & 72 & 55 \\
\hline Linguagem AND HD & 3 & 1 & 1047 & 69 & 9 \\
\hline Matemática AND HD & 0 & 0 & 5 & - & 3 \\
\hline Memória AND HD & 5 & 0 & 750 & 81 & 16 \\
\hline Orientação têmporo-espacial AND HD & 0 & 0 & 2 & - & 1 \\
\hline Percepção AND HD & 3 & 0 & 584 & 47 & 23 \\
\hline Total & 25 & 6 & 4236 & 435 & 169 \\
\hline
\end{tabular}

Observa-se, na Tabela 3, que as alterações mais associadas à LHD do total de estudos mantidos na quantificação final do LILACS, foram heminegligência (50\%), comunicação (33\%) e linguagem (33\%). Do MEDLINE foram mantidas as publicações sobre as seguintes alterações, em ordem decrescente: heminegligência (32\%); atenção (15\%); percepção (14\%); anosognosia (12\%); alterações da memória (9\%); linguagem (5\%); do comportamento (4\%); funções executivas (3\%); emoção (2\%); alterações da comunicação (1\%); orientação têmporo-espacial (1\%); habilidades aritméticas $(0 \%)$. Agrupando-se as alterações emocionais com as comportamentais, ambas consideradas déficits do processamento da emoção, obtém-se $6 \%$ dos estudos. A soma dos estudos de comunicação e de linguagem totaliza $6 \%$.

\section{DISCUSSÃO}

$\mathrm{O} \mathrm{HE}$ e as funções a ele relacionadas começaram a ser estudados um século antes do HD, o que justifica a disparidade no número de estudos sobre o papel de cada um dos hemisférios (para uma revisão, ver Brookshire, 2003; Lezak, Howieson, \& Loring, 2004). Apesar do total de estudos acerca do HD ainda ser pouco numeroso, percebe-se, atualmente, um redirecionamento no foco dos trabalhos, havendo um crescente interesse quanto às funções desse hemisfério, investigadas em populações saudáveis (por exemplo, Coulson \& Williams, 2005; Laeng, Overvoll, \& Steinsvik, 2007) e neurológicas (por exemplo, Zaidel, Kasher, Soroker, \& Batori, 2002).

No Brasil, entretanto, a carência de estudos sobre esse tema é ainda maior do que no contexto internacional, o que foi comprovado no presente estudo pela comparação entre a quantidade de pesquisas encontradas na base LILACS e na MEDLINE. A ausência de investigações na literatura nacional pesquisada pode estar ligada ao restrito conhecimento sobre as especializações do HD, assim como à falta de instrumentos de avaliação neuropsicológica adequados à nossa realidade sócio-linguístico-cultural. Recentemente, alguns esforços têm sido feitos no sentido de suprir essa demanda quanto à avaliação das habilidades relacionadas ao HD. A exemplo disso, pode se mencionar a adaptação da Bateria Montreal de Avaliação da Comunicação - Bateria MAC (Fonseca, Parente, Côté, Ska, \& Joanette, 2007). Esse instrumento canadense é o primeiro que se propõe a analisar quatro habilidades de processamento comunicativo, geralmente prejudicadas em indivíduos com LHD, a saber: discursiva, pragmático-inferencial, léxico-semântica e prosódica.

Apesar de terem sido encontrados 31 estudos internacionais, número evidentemente maior do que no 
contexto nacional, essa quantidade pode ser considerada ainda pouco significativa, uma vez que as alterações comunicativo-cognitivo-comportamentais pósAVC de HD e as sequelas de LHD em geral ainda não são suficientemente conhecidas, sendo escassas as investigações teóricas e empíricas até mesmo em nível internacional. Quanto aos resultados referentes à distribuição das investigações internacionais por tipo e objeto de pesquisa, foram encontrados mais estudos empíricos do que teóricos. Essa diferença era esperada tendo-se em vista o crescente interesse pelo papel do HD nas duas últimas décadas (Van Lancker, 1997).

Dentre os estudos empíricos, a quantidade maior de estudos de grupo encontrada frente a uma menor quantidade de investigações do tipo estudo de caso pode justificar, pelo menos em parte, a heterogeneidade de manifestação das alterações comunicativocognitivo-comportamentais decorrentes de LHD referida na literatura (Brookshire, 2003; Myers, 1999; Tompkins e cols., 2002). Os estudos de grupo, no caso da LHD e da investigação neuropsicológica como um todo, mostram-se demasiadamente limitados, pois, dadas as acentuadas diferenças inter-sujeito na manifestação dos sintomas dela decorrentes, é difícil estabelecer critérios de inclusão válidos para todos os pacientes. Além disso, nos estudos de grupo, os escores agrupados em médias mascaram os desempenhos individuais (Willmes, 1998). Assim, surge uma dupla dificuldade, na medida em que, ao se ampliar os critérios de inclusão para abarcar uma maior variabilidade de sintomas, pode-se diminuir o controle de variáveis intervenientes, tais como, diferentes atributos da lesão (extensão, profundidade e tempo pós-lesão) e distintas capacidades pré-mórbidas (qualidade da escolarização, nível sociocultural etc). Com essa diminuição do rigor metodológico na seleção das amostras clínicas, corre-se o risco de acabar incluindo-se sujeitos com diferentes perfis de manifestação da "SHD", devido à associação do efeito da lesão propriamente dita à influência de variáveis demográficas, neurológicas, entre outras. Nesse caso, a ocorrência de falsos positivos também aumenta, já que indivíduos com dificuldades cognitivas devido a outras variáveis, tais como idade avançada ou baixa escolaridade, podem apresentar os mesmos sintomas das alterações pós-AVC de HD não decorrentes da lesão, mas sim dessas variáveis sociodemográficas. Por outro lado, um exagerado refinamento nos critérios de inclusão de pacientes pode levar a uma redução importante do tamanho amostral, o que poderia ocasionar a exclusão de alguns indivíduos com LHD com diferentes perfis clíni- cos (Flyvbjerg, 2004; Siksou, 2005), gerando falsos negativos.

Apesar da importância dos estudos de grupo para comparações entre populações neurologicamente preservadas e populações neurológicas ou diferentes populações neurológicas entre si, os estudos de caso são considerados muito importantes para o avanço dos conhecimentos de neuropsicologia (Willmes, 1998). Esse último delineamento é mais interessante na medida em que possibilita a compreensão de certas nuances que poderiam não ser percebidas em estudos de grupo. A partir de estudos de caso, pode-se detectar a dissociação entre diferentes processamentos, por exemplo. Mediante um estudo de caso individual, uma dissociação simples poderia ser averiguada, tal como entre habilidades linguísticas sintáticas preservadas e habilidades comunicativas pragmáticas prejudicadas. Em complementaridade, um estudo de casos múltiplos permitiria a pesquisa de dissociações duplas, com padrões inversos entre dois ou mais casos. Além do mais, estudos de casos viabilizam corroborar ou refutar hipóteses previamente levantadas em estudos de grupo, bem como possibilitam uma melhor compreensão da síndrome (Siksou, 2005; Flyvbjerg, 2004). Contudo, convém ressaltar que ambas as abordagens apresentam limitações (Kristensen, Almeida, \& Gomes, 2001). Uma forma de minimizá-las seria a utilização de estudos de grupo com análise de clusters (agrupamentos), possibilitando a delimitação de subgrupos clínicos com diferentes manifestações de uma neuropsicopatologia, segundo as características dos participantes (Côté, Payer, Giroux, \& Joanette, 2007; Salles, 2005). Dentro de cada agrupamento, os casos poderiam ser estudados com maior profundidade.

Além das dificuldades referentes às questões metodológicas, agregam-se limitações conceituais no estudo das alterações comunicativo-cognitivo-comportamentais pós-AVC de HD que também foram nominadas de Síndrome do HD. Encontra-se uma vasta amplitude de termos referindo-se a esse quadro. A falta de uma terminologia consistente gera uma série de dificuldades para que ela seja investigada. A heterogeneidade dos termos cria a impossibilidade de uma definição precisa e, consequentemente, impede o intercâmbio entre diferentes disciplinas e mesmo entre pesquisadores de uma mesma área de conhecimento. Ao mesmo tempo, traz dificuldades para a prática do diagnóstico e da reabilitação de pacientes acometidos por LHD. Por outro lado, a falta de uniformidade quanto à terminologia utilizada retrata a ampla variedade de sinais e sintomas presentes nesse quadro (Blake e cols., 2003). 
Ademais, associados à variabilidade de nomenclatura, estão os diferentes enfoques na formação dos profissionais que atuam em neuropsicologia, implicando vieses quanto à identificação dos déficits das funções cognitivas, comunicativas e emocionais prejudicadas. Então, um profissional de uma determinada área pode detectar sinais que passariam despercebidos por um clínico de outra especialidade. Os sintomas tratados, pois, serão aqueles detectados pelo profissional, e não necessariamente abarcarão a totalidade dos prejuízos apresentados pelo paciente (Blake e cols., 2003).

Associada às dificuldades metodológicas e de nomenclatura das alterações comunicativo-cognitivocomportamentais na LHD, encontra-se uma ampla variabilidade de sinais e sintomas inerentes a essa neuropsicopatologia. $\mathrm{O}$ termo síndrome refere-se ao conjunto de sinais e sintomas relacionados a qualquer processo mórbido, e que formam juntos o quadro da doença (Stedman Dicionário Médico, 1996). Com base nessa definição, entende-se o porquê do termo Síndrome do HD não ser utilizado de modo consensual pelos pesquisadores e clínicos, uma vez que muitos de seus sintomas ainda não são conhecidos pela comunidade científica e não necessariamente estão presentes em todos os pacientes com LHD. Em função dessa multiplicidade de sinais pós-AVC de HD, são necessários mais estudos para que se desenvolva uma delimitação de seus diferentes subtipos e perfis. Uma das sugestões apresentadas na literatura para refletir sobre o desafio do termo clínico dos distúrbios comunicativos verbais pós-LHD é "afasia do HD" (Joanette e cols., 2007).

Em relação à terceira questão de pesquisa, nota-se que nos estudos das categorias inicial e final, de acordo com a Tabela 3, dentre todos os déficits cognitivos, emocionais e comunicativos relatados como fazendo parte da de um quadro pós-AVC de HD ou associados a LHD, a heminegligência é abordada em uma frequência considerável na literatura. Esse sintoma, inclusive, chega a ser considerado como uma síndrome independente por alguns autores (Rode e cols., 2005; Verfaellie \& Heilman, 2006).

A heminegligência manifesta-se geralmente pela não resposta do paciente a estímulos sensoriais - táteis, visuais e/ou auditivos - provenientes do hemiespaço contralateral ao hemisfério lesado (Hommet e cols., 2004). A revisão dos conceitos desse quadro demonstra que não há consenso na literatura em relação à função cognitiva deficitária na heminegligência: há autores que defendem a hipótese de que se trata de distúrbios atencionais (Deouell, Sacher, \& Soroker,
2005; Verfaellie \& Heilman, 2006), enquanto outros defendem que são decorrentes de déficits perceptivos (por exemplo, Lu, Ye, Zhou, Lu, \& Chen, 2005). Esses diferentes enfoques teóricos podem gerar uma ambiguidade quando se trata de definir qual a função cognitiva mais abordada nos estudos sobre a "SHD". De um lado, considerando-se a heminegligência como um déficit atencional, a atenção, consequentemente, seria considerada a habilidade mais abordada. Somando-se o total de estudos sobre heminegligência aos que abordam a atenção, ter-se-ia, portanto, um total de $65 \%$ dos estudos encontrados. Por outro lado, realizando-se o mesmo processo com a percepção, chegase a resultado próximo (64\%). A heminegligência é geralmente acompanhada por anosognosia, que corresponde à ausência de consciência plena dos déficits, ou ainda da amplitude do impacto funcional desses na vida do indivíduo lesado (Joanette, Ska, \& Côté, 2004; Rode e cols, 2005). Da mesma forma que a heminegligência, esse distúrbio também pode estar associado a déficits perceptivos (Bryan, 1995) ou atencionais (Heilman, Barrett, \& Adair, 1998). Frente à ausência de unanimidade na literatura quanto à função afetada, o ideal é que os quadros sejam agrupados às duas funções.

A grande frequência de estudos que relacionam a heminegligência aos acometimentos do HD deve-se ao fato de que esta síndrome é muito prevalente após uma lesão desse hemisfério. Na comparação da quantidade de pacientes com LHD e com lesão de HE (LHE) que apresentam heminegligência, Deouell, Sacher e Soroker (2005) constataram que $66 \%$ dos pacientes com LHD avaliados tiveram déficits no hemicampo contralateral; em contrapartida, apenas $31 \%$ dos indivíduos com LHE apresentavam esse quadro. Essa alteração também é bastante enfocada em função dos prejuízos causados à rotina diária do indivíduo com LHD não somente pelo próprio déficit, mas também porque nos casos em que estão presentes, há um agravamento dos demais sintomas, aumentando a severidade das dificuldades neuropsicológicas. Do mesmo modo, a anosognosia mostra-se também significativamente mais frequente em indivíduos com LHD do que em indivíduos com LHE (Buxbaum e cols., 2004).

O agrupamento dos estudos nas três grandes áreas de alteração que podem ocorrer - cognição, comunicação e emoção - permite constatar que os estudos acerca das alterações cognitivas são a maioria. Os estudos sobre os aspectos comunicativos e linguísticos mostram um redirecionamento do enfoque tradicional 
no papel do HE no processamento linguístico para o papel do HD juntamente com o HE no desempenho das habilidades comunicativas. Os ganhos desse redirecionamento estendem-se para os contextos de avaliação da comunicação (Bryan, 1995) e da reabilitação (Brookshire, 2003).

O número restrito de publicações comunicação em relação aos relacionados à comunicação já era esperado na medida em que a lacuna de instrumentos para avaliação de componentes comunicativos pode ter contribuído para restringir pesquisas que visassem examinar este processamento após AVC. Ademais, é importante ressaltar que as dificuldades comunicativas, considerando os processamentos prosódico, léxico-semântico, discursivo e pragmático-inferencial, podem afetar um ou mais destes componentes, de modo heterogêneo. Assim, seria necessário desenvolver investigações que examinassem todos estes em um mesmo protocolo de avaliação.

Em suma, observa-se que há uma predominância de estudos acerca dos déficits cognitivos, associados à LHD, seguidos pelas alterações comunicativas e, por fim, pelas emocionais. Tal achado evidencia que, embora comumente seja atribuído ao HD a função de processamento emocional, atualmente também tem sido atribuído a esse hemisfério importante participação no desempenho das funções cognitivas, bem como comunicativas.

\section{CONSIDERAÇÕES FINAIS}

A partir dessa investigação, constatou-se que há heterogeneidade quanto à nomenclatura e às manifestações das alterações comunicativo-cognitivo-comportamentais decorrentes de AVC de HD, sendo, portanto, necessários estudos teóricos para consolidar o corpo de conhecimento sobre esse quadro. Os estudos de grupo com análise de clusters são importantes para uma melhor caracterização desse quadro, como também para a delimitação de seus diferentes subtipos. Estudos de caso também podem auxiliar no entendimento das associações/dissociações entre as diferentes funções afetadas nessa síndrome.

Com base na reduzida quantidade de estudos nacionais e internacionais sobre essa neuropsicopatologia, mostra-se fundamental a continuidade de estudos e reflexões das sequelas neuropsicológicas pós-LHD. A difusão do corpo teórico sobre esse tema é indispensável para que os clínicos possam fazer seu diagnóstico e elaborar um plano de reabilitação mais acurado.

Por fim, ressalta-se que, embora o papel do HD esteja sendo evidenciado na literatura, é importante ter- se o cuidado de não defender arduamente o extremo oposto do tradicional conceito de dominância cerebral. A participação do HD não é a única evidenciada durante tarefas de processamento cognitivo, comunicativo e emocional. Há, em geral, uma cooperação inter e intrahemisférica em tais processamentos.

\section{REFERÊNCIAS}

Allegri, R. F., Elli, J., Valicenti, M. R., Mangone, C. A., Taragano, F. E., \& Ranalli, C. G. (1996). Síndromes neuropsiquiátricos por lesión en el hemisferio cerebral derecho. Acta Psiquiátrica y Psicológica de América Latina, 42, 222229.

Annoni, J. M., Khateb, A., Gramigna, S., Staub, F., Carota, A., Maeder, P., \& Bogousslavsky, J. (2003). Chronic cognitive impairment following laterothalamic infarcts: A study of 9 cases. Archives of Neurology, 60, 1439-1443.

Benedet, M. J. (1995). Origen y evolución de la neuropsicología y de sus aportaciones al daño cerebral traumático. Em M. J. Benedet, Daño cerebral traumático, neuropsicología y calidad de vida (pp. 117-144). Madrid: Fundación MAPFRE Medicina.

Blake, M. L., Duffy, J. R., Tompkins, C. A., \& Myers, P. S. (2003). Right hemisphere syndrome is in the eye of the beholder. Aphasiology, 17, 423-432.

Bouaffre, S., \& Faïta-Ainseba, F. (2007). Hemispheric differences in the time-course of semantic priming processes: Evidence from event-related potentials (ERPs). Brain and Cognition, 63, 123-135.

Brookshire, R. H. (2003). Introduction to neurogenic communication disorders. Saint Loius, MO: Mosby.

Bryan, K. L. (1995). The right hemisphere language battery $\left(2^{\mathrm{a}}\right.$ ed.). London: Whurr.

Burns, M. S., Halper, A. S., \& Mogil, S. I. (1985). Clinical management of right hemisphere dysfunction. Chicago, IL: Aspen.

Buxbaum L. J., Ferraro M. K., Veramonti T., Farne A., Whyte J., Ladavas E., Frassinetti F., \& Coslett H. B. (2004). Hemispatial neglect: Subtypes, neuroanatomy, and disability. Neurology, 62, 749-756.

Code, C. (1987). Language aphasia and the right hemisphere. Chichester, Enlgand: J. Wiley.

Côté, H., Payer, M., Giroux, F., \& Joanette, Y. (2007). Towards a description of clinical communication impairment profiles following right-hemisphere damage. Aphasiology, 21, 739-749.

Coulson, S., \& Williams, R. F. (2005). Hemispheric asymmetries and joke comprehension. Neuropsychologia, 43, 128-141.

Deouell L. Y., Sacher Y., \& Soroker N. (2005). Assessment of spatial attention after brain damage with a dynamic reaction time test. Journal of the International Neuropsychological Society, 11, 697-707.

Eisenson, J. (1959). Language dysfunctions associated with right brain damage. American Speech and Hearing Association, 1, 107.

Fonseca, R. P., \& Parente, M. A. M. P. (2007) Metanálise de estudos do processamento comunicativo em indivíduos com lesão vascular direita. Estudos de Psicologia, 24, 529-538.

Fonseca, R. P., Parente, M. A. M. P., Côté, H., Ska, B., \& Joanette, Y. (2008). Bateria Montreal de avaliação da comunicação Bateria MAC. Barueri: Pró-Fono. 
Flyvbjerg, B. (2004). Five misunderstandings about case-study research. Em C. Seale, G. Gobo, J. F. Gubrium, \& D. Silverman (Orgs.), Qualitative research practice (pp. 420-434). London: Sage.

Heilman, K. M., Barrett, A. M., \& Adair, J. C. (1998). Possible mechanisms of anosognosia: A defect in self-awareness. Philosophical Transactions of the Royal Society of London Biological Sciences, 353, 1903-1909.

Hommet, C., Bardet, F., de Toffol, B., Perrier, D., Biraben, A., Vignal, J.P., Scarabin, J.M., Corbineau, M., \& Chauvel, P. (2004). Unilateral spatial neglect following right inferior parietal cortectomy. Epilepsy \& Behavior, 5, 416-419.

Joanette, Y., Côté, H., Fonseca, R. P., Giroux, F., Mejia-Constain B., \& Ska, B. (2007). Quando os hemisférios direito e esquerdo colaboram: A dinâmica inter-hemisférica subjacente à linguagem e suas implicações para a prática clínica e para o envelhecimento bem-sucedido. Em E. C. Macedo, L. I. Z. Mendonça, A. F. Veloso, B. B. G. Schlecht, K. Z. Ortiz, \& D. A. Azambuja (Orgs.), Avanços em neuropsicologia: Das pesquisas à aplicação clínica (pp. 17-31). São Paulo: Livraria Santos.

Joanette, Y., Goulet, P., \& Hannequin, D. (1990). Right hemisphere and verbal communication. New York, NY: Springer.

Joanette, Y., Ska, B., \& Côté, H. (2004). Protocole MEC Protocole Montreál d'évaluation de la communication. Montréal: Ortho Édition.

Kristensen, C. H., Almeida, R. M. M., \& Gomes, W. B. (2001). Desenvolvimento histórico e fundamentos metodológicos da neuropsicologia cognitiva. Psicologia: Reflexão e Crítica, 14, 259-274.

Kumral, E., \& Evyapan, D. (2000). Reversed clock phenomenon: A right-hemisphere syndrome. Neurology, 55, 151-152.

Laeng, B., Overvoll, M., \& Steinsvik, O. O. (2007). Remembering 1500 pictures: The right hemisphere remembers better than the left. Brain and Cognition, 63, 136-144.

Landau, Y. E., Auerbach, J. G., Gross-Tsur, V., \& Shalev, R. S. (2003). Speed of performance of children with developmental right hemisphere syndrome and with attention-deficit hyperactivity disorder. Journal of Child Neurology, 18, 264-268.

Lezak, M. D., Howieson, D. B., \& Loring, D. W. (2004). Neuropsychological assessment. New York, NY: Oxford University Press.

Lu, X. S., Ye, J., Zhou, S., Lu, B. X., \& Chen, X. H. (2005). Unilateral spatial neglect, global processing deficit and prosopagnosia following right hemisphere stroke: A case report. Chinese Medical Journal, 118, 1846-1848.

Marchetti, C., Carey, D., \& Della Sala, S. (2005). Crossed right hemisphere syndrome following left thalamic stroke. Journal of Neurology, 252, 403-411.

Martins, I. P., Antunes, N. L., Castro-Caldas, A., \& Antunes, J. L. (1995). Atypical dominance for language in developmental dysphasia. Developmental Medicine \& Child Neurology, 37, 85-90.
Morin, C., Taillefer, C., Vallat, C., Helsly, N., Thibierge, S., \& Pradat-Diehl, P. (2001). Qu'est-ce qu'un gauche? Annales de Réadaptation et de Médecine Physique, 44, 192-204.

Myers, P. S. (1999). Right hemisphere damage: Disorders of communication and cognition. San Diego, CA: Singular.

Myers, P. S. (2001). Toward a definition of RHD syndrome. Aphasiology, 15, 913-918.

Pimental, P. A., \& Kingsbury, N. A. (1989). Mini inventory of right brain injury. Austin, TX: PRO-ED.

Rode, G., Thomas-Antérion, C., Luauté, J., Jacquin-Courtois, S., Ciancia, S., Rossetti, Y., \& Boisson, D. (2005). Évaluation des incapacités et de la qualité de vie des patients présentant des troubles cognitifs. Annales de Réadaptation et de Médicine Physique, 48, 376-391.

Salles, J. F. (2005). Habilidades e dificuldades de leitura e escrita em crianças de $2^{a}$ série: Abordagem neuropsicológica cogniti$v a$. Tese de Doutorado não-publicada, Universidade Federal do Rio Grande do Sul. Porto Alegre, RS.

Siksou, M. (2005). Introduction à la neuropsychologie. Paris: Dunod.

Stedaman, T. L. (1996). Stedman dicionário médico (S. A. Teixeira, Trad.). Rio de Janeiro: Guanabara Koogan.

Tompkins, C. A. (1995). Right hemisphere communication disorders: Theory and management. San Diego, CA: Singular.

Tompkins, C. A, Fassbinder, W., Lehman-Blake, M. T., \& Baumgaertner, A. (2002). The nature and implications of right hemisphere language disorders: Issues in search of answer. Em A. E. Hillis (Org.), The handbook of adult language disorders Integrating cognitive neuropsychology, neurology, and rehabilitation (pp. 429-448). New York, NY: Psychology Press.

Van Lancker, D. V. (1997). Rags to riches: Our increasing appreciation of cognitive and communicative abilities of the human right cerebral hemisphere. Brain and Language, 57, 1-11.

Verfaellie, M., \& Heilman, K. M. (2006). Neglect syndromes. Em P. J. Snyder, P. D. Nussbaum., \& D. L. Robins (Orgs.), Clinical neuropsychology (pp. 489-507). Washington, DC: American Psychological Association.

Willmes, K. (1998). Methodological and statistical considerations in cognitive neurolinguistics. Em B. Stemmer \& H. A. Whitaker (Orgs.), Handbook of neurolinguistics (pp. 57-70). San Diego, CA: Academic Press.

Zaidel, E., Kasher, A., Soroker, N., \& Batori, G. (2002). Effects of right and left hemisphere damage on performance of the "Right Hemisphere Communication Battery". Brain and Language, $80,510-535$.
Recebido: $21 / 02 / 2007$

Última Revisão: 20/03/2010 Aceito: $30 / 04 / 2010$ 